\title{
Human T-lymphotropic virus type 2 subtype $b$ in a patient with chronic neurological disorder
}

\author{
Carolina Rosadas • Ana C. P. Vicente $\cdot$ Louise Zanella • \\ Mauro J. Cabral-Castro • José M. Peralta • \\ Marzia Puccioni-Sohler
}

Received: 1 June 2014 / Revised: 28 July 2014 / Accepted: 6 August 2014 / Published online: 3 September 2014

(C) Journal of NeuroVirology, Inc. 2014

\section{Introduction}

Human T-cell lymphotropic virus is a retrovirus described worldwide. There are four HTLV types: HTLV-1 to 4. Only HTLV-1 and 2 are linked to human disease. The majority of infected individuals remain asymptomatic. However, it is estimated that $5 \%$ of HTLV-1-infected patients present a clinical neurological disorder called HTLV-1-associated myelopathy (HAM/TSP) (Osame 1990). The diagnosis of HAM/ TSP is made according to WHO criteria. The presence of specific antibodies in both blood and CSF, associated with slowly progressive spastic paraparesis, characterizes HAM/ TSP (Osame 1990; Puccioni-Sohler et al. 2001). HTLV-1 infection has also been related with others neurologic manifestations, such as sensory neuropathy, cognitive deficit, myositis, erectile dysfunctions, and isolated bladder dysfunction. These rare clinical presentations may be associated with HAM/TSP (Biswas et al. 2009). HTLV-2 is endemic among

C. Rosadas $\cdot$ M. J. Cabral-Castro $\cdot$ J. M. Peralta $\cdot$ M. Puccioni-Sohler Universidade Federal do Rio de Janeiro (UFRJ), Rio de Janeiro, Brazil

A. C. P. Vicente $\cdot$ L. Zanella

Laboratory of Molecular Genetics of Microorganisms, Fundação

Oswaldo Cruz (IOC-FIOCRUZ), Rio de Janeiro, Brazil

M. Puccioni-Sohler

Universidade Federal do Estado do Rio de Janeiro (UNIRIO), Rio de Janeiro, Brazil

C. Rosadas $(\bowtie) \cdot$ M. J. Cabral-Castro $\cdot$ M. Puccioni-Sohler $(\bowtie)$ Faculdade de Medicina, Universidade Federal do Rio de Janeiro (UFRJ), Laboratório de Líquido Cefalorraquiano, SPC, Hospital Universitário Clementino Fraga Filho, (HUCFF/UFRJ), Rua Professor Rodolpho Paulo Rocco 255, 3 andar, 21941-913 Rio de Janeiro, RJ, Brazil

e-mail: carolrosadas@gmail.com

e-mail: mpuccioni@hucff.ufrj.br
Amerindians and has been reported at a high prevalence in intravenous drug abusers in the USA, Europe, and Asia (Araujo and Hall 2004; Morimoto et al. 2007). The presence of disease in HTLV-2 infection is rare. Previous studies reported an association between HTLV-2 infection and the development of a clinical manifestation similar to HAM/TSP as well as sensory neuropathy (Harrington et al. 1993; Jacobson et al. 1993; Lehky et al. 1996; Araujo and Hall 2004; Biswas et al. 2009). Four genetic HTLV-2 subtypes (a, $\mathrm{b}, \mathrm{c}$, and d) have been described. All documented cases of HTLV-2 and neurological disturbances are associated with HTLV-2a (Harrington et al. 1993; Jacobson et al. 1993; Lehky et al. 1996; Araujo and Hall 2004). The present study reports the first case of HAM/TSP-like illness caused by HTLV-2b, in a patient from Brazil. This patient was also coinfected with hepatitis $\mathrm{C}$ virus (HCV).

\section{Case report}

A 53-year-old white man from Rio de Janeiro, Brazil, reported a 5-year history of lower limb weakness and urinary incontinence. He had history of treatment for chronic HCV infection and intravenous drug use. Neurological examination revealed spastic paraparesis, hyperreflexia of the lower limbs, and bilateral Babinski sign. Routine blood tests, folic acid, vitamin B12, rheumatoid factor, antinuclear factor (ANF), LE cells, Waaler Rose, and thyroid hormones (TSH, T3, and T4) concentration were normal, but for reactive serology for $\mathrm{HCV}$. Serologic test for syphilis (VDRL) was negative. The presence of HTLV-1/2 antibody in serum was evaluated by ELISA (Diasorin, UK). HTLV-2 infection was confirmed by discriminatory Western blot (GD21,p29, p36, and Rgp46-II). The tax gene was recovered by PCR and sequenced to confirm HTLV type. The predict amino acid 


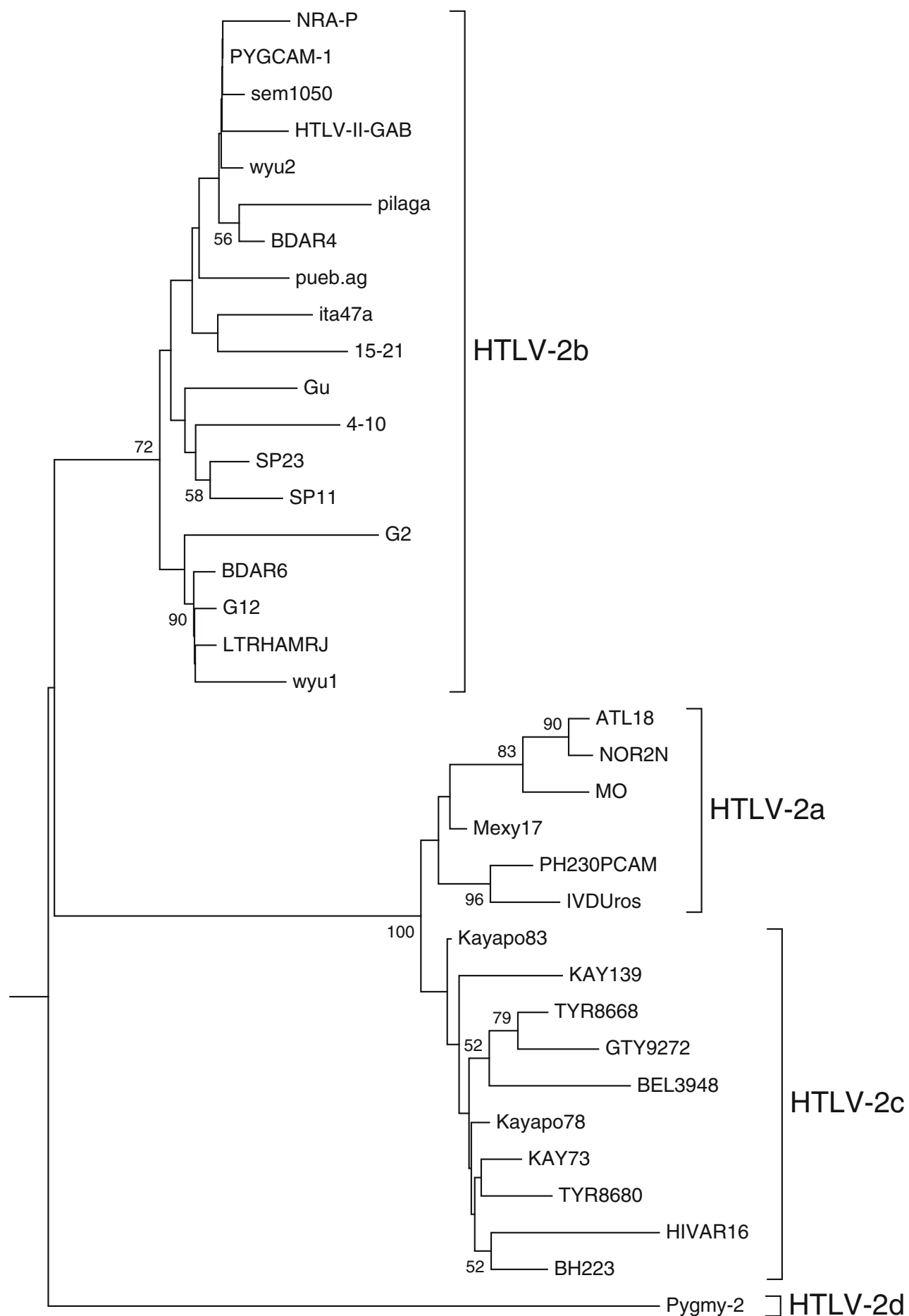

\subsection{5}

Fig. 1 Genetic Analysis of 653-bp LTR sequence from infected subject (LTRHAMRJ) and HTLV-2 reference sequences. The neighbor-joining tree was inferred under a Tamura-3 parameter evolutionary model and gamma-distribution. Bootstrap values using 1,000 replicates. The accession numbers of GenBank sequences are NRA (L20734); PYGCAM-1 (Z46888); Sem 1050 (U10263); ATL 18 (U10252); Ita47a (U10254); NORN2N (U10258); PUEB.AG (U10261); WYU1 (U12792); Wyu2 (U12794); 4-10 (U73016); 15-21 (U73017); SP23 (AY442386); Sp11
(AY442374);G2 (AF074965); G12 (L11456); Kayapo83 (AF139390); HTLV-II-GAB (Y13051); MO (M10060); Mexy17 (L42510); PH230PCAM (Z46838); IVDUros (AF054272); TYR8668 (AF306729); GTY9272 (AF306727); BEL3948 (AF306724); Kayapo78 (AF139388); Kay73 (L42509); TYR8680 (AF306730); HIVAR16 (JN222964); BH223 (AY509600); Kay139 (L42508); Pilaga (AF054271); BDAR4 (JN222945); BDAR6 (JN222947); Pygmy-2 (Y14365); GU (X89270) 
sequence revealed that the provirus presented an extended Tax, characteristic of HTLV-2b. LTR sequence was obtained and the phylogenetic analysis confirmed the HTLV-2 subtype b (Fig. 1). The tax and LTR sequences accession numbers in GenBank are KJ826466 and KJ826465, respectively. HTLV-2 relative quantification was evaluated using real-time PCR targeting the $\operatorname{tax}$ gene. The proviral load was 39 times higher in the present patient sample when compared with other HTLV-2 symptomatic patient (Silva et al. 2002) (2-[delta][delta]Ct=5,27).

The cerebrospinal fluid (CSF) was collected by lumbar puncture. Total and specific cellular count, glucose, and total protein were normal. Bacterial, fungal, and mycobacterial cultures of CSF were negative as also the immunological test for syphilis. The presence of HTLV-1/2 antibodies in CSF was detected by ELISA. Blood-cerebrospinal barrier dysfunction was not observed. There was no intrathecal synthesis of total IgG (by IgG Index).

Magnetic resonance of brain and dorsal spine, electroneuromyography, and somatosensory evoked potentials were also normal.

The study was approved by the ethics committees of the HUGG/UNIRIO and HUCFF/UFRJ. The patient signed the consent inform.

\section{Discussion}

This study unequivocally showed the presence of HTLV$2 \mathrm{~b}$ infection in a case of chronic spastic paraparesis, clinically indistinguishable from HAM/TSP. This is the first description of HAM/TSP-like illness caused by this HTLV-2 subtype that had not been identified before in Rio de Janeiro/Brazil.

Jacobson et al. (1993) reported the first case of HTLV-2 infection associated with neurological disorder (Jacobson et al. 1993). However, even with some countries presenting high prevalence of infections due to this HTLV type, there are only a few reports concerning neurological manifestations in HTLV-2 infection (Jacobson et al. 1993; Lehky et al. 1996; Araujo and Hall 2004).

HTLV co-infections are not rare particularly in some risk groups (Araujo and Hall 2004). In our report, the patient presented $\mathrm{HCV}$ chronic infection. $\mathrm{HCV}$ can result in some extrahepatic manifestation (EHM) such as neuropathy, whose immunopathological mechanisms are related to symptomatic disease. In our study, neuropathy was excluded by normal electroneuromyography. Furthermore, he did not present any evidence of an autoimmune disorder.

The patient presented normal CSF, but for the presence of HTLV-1/2 antibodies. This finding has also been observed in the majority of HTLV-2-infected individuals with neurological alterations. Increased protein concentration and intrathecal synthesis of antibodies are rarely reported (Lehky et al. 1996; Araujo and Hall 2004) was not observed.

It is well established that the proviral load influences the disease progression in HTLV-1-infected individuals (Murphy et al. 2004; Silva et al. 2007; Melamed et al. 2013; Rosadas et al. 2013). In this context, Murphy et al. (2004) showed that the proviral load is higher in HTLV-1 than in HTLV-2-infected individuals. This could explain why disease progression is most commonly associated with HTLV-1 infection (Murphy et al. 2004). HTLV-1 and HTLV-2 present different forms of regulatory Tax protein that seems to be related with the viral pathogenesis. Higuchi and Fujii (2009) described that HTLV1 and HTLV-2-infected T-cells grow equivalently in environments with high concentration of T-cell growth-promoting cytokines (such as interleukin-2). However, under conditions of low levels of these cytokines, as is observed in vivo, HTLV1-infected cells can grow more efficiently than HTLV-2infected T-cells. This difference may be linked with the diversity of Tax protein in these viral types. This would result in an increased expansion of HTLV-1-infected T-cell and a high proviral load (Higuchi and Fujii 2009).

Regarding the HTLV-2 subtypes, to date, only HTLV$2 \mathrm{a}$ has been associated with neurological disorders. Murphy et al. (2004) demonstrated that HTLV-2ainfected individuals presented a high proviral load when compared to those with HTLV-2b. This could lead to more severe infection caused by HTLV-2a, which may determine its association with disease. Interestingly, it was previously reported that $\mathrm{HCV}$-infected patients show higher levels of circulating cytokines, such as interleukin-2, than non-infected individuals (Sofian et al. 2012). By hypothesis, the HCV co-infection could be contributing to the proliferation of HTLV-2b-infected T-cells in the reported case. Lehky et al. (1996) observed that three of four patients with neurological disorder associated with HTLV-2a were co-infected with $\mathrm{HCV}$.

In fact, the proviral load of the studied patient was higher than other HTLV-2a symptomatic patient that was previously described (Silva et al. 2002).

These findings confirm that HTLV-2b may be related to neurological disorders and serve as an alert to the possible role of HCV co-infection in the neuropathogenesis of HTLV-2 infection.

Acknowledgments To PDTIS/FIOCRUZ sequencing platform.

This work was supported by Fundação de Amparo a Pesquisa do Estado do Rio de Janeiro (FAPERJ) and by Post-Graduation on Infectious and Parasitic Diseases (UFRJ), a PhD fellowship to C.R. from Coordenação de Aperfeiçoamento de Pessoal de Nível Superior (CAPES), and a PhD fellowship to M.J.C.C. and L.Z. from Conselho Nacional de Desenvolvimento Científico e Tecnológico (CNPq). 
Conflict of interests The authors declare that they have no conflict of interest.

\section{References}

Araujo A, Hall WW (2004) Human T-lymphotropic virus type II and neurological disease. Ann Neurol 56:10-19. doi:10.1002/ana.20126

Biswas HH, Engstrom JW, Kaidarova Z et al (2009) Neurologic abnormalities in HTLV-I- and HTLV-II-infected individuals without overt myelopathy. Neurology 73:781-789. doi:10.1212/WNL. 0b013e3181b6bba9

Harrington WJ, Sheremata W, Hjelle B et al (1993) Spastic ataxia associated with human T-cell lymphotropic virus type II infection. Ann Neurol 33:411-414. doi:10.1002/ana.410330416

Higuchi M, Fujii M (2009) Distinct functions of HTLV-1 Tax1 from HTLV-2 Tax 2 contribute key roles to viral pathogenesis. Retrovirology 6:117. doi:10.1186/1742-4690-6-117

Jacobson S, Lehky T, Nishimura M et al (1993) Isolation of HTLV-II from a patient with chronic, progressive neurological disease clinically indistinguishable from HTLV-I-associated myelopathy/tropical spastic paraparesis. Ann Neurol 33:392-396. doi:10.1002/ana.410330411

Lehky TJ, Flerlage N, Katz D et al (1996) Human T-cell lymphotropic virus type II-associated myelopathy: clinical and immunologic profiles. Ann Neurol 40:714-723. doi:10.1002/ana.410400507

Melamed A, Laydon DJ, Gillet NA et al (2013) Genome-wide determinants of proviral targeting, clonal abundance and expression in natural HTLV-1 infection. PLoS Pathog 9:e1003271. doi:10.1371/ journal.ppat.1003271
Morimoto HK, Morimoto AA, Reiche EMV et al (2007) Difficulties in the diagnosis of HTLV-2 infection in HIV/AIDS patients from Brazil: Comparative performances of serologic and molecular assays, and detection of HTLV-2b subtype. Rev Inst Med Trop Sao Paulo 49:225-230

Murphy EL, Lee T-H, Chafets D et al (2004) Higher human T lymphotropic virus (HTLV) provirus load is associated with HTLV-I versus HTLV-II, with HTLV-II subtype A versus B, and with male sex and a history of blood transfusion. J Infect Dis 190: 504-510. doi:10.1086/422398

Osame M (1990) Review of WHO Kagoshima meeting and diagnostic guidelines for HAM/TSP. Hum Retrovirol HTLV 191-197

Puccioni-Sohler M, Rios M, Carvalho SM et al (2001) Diagnosis of HAM/TSP based on CSF proviral HTLV-I DNA and HTLV-I antibody index. Neurology 57:725-727

Rosadas C, Cabral-Castro MJ, Vicente ACP et al (2013) Validation of a quantitative real-time PCR assay for HTLV-1 proviral load in peripheral blood mononuclear cells. J Virol Methods 193:536-541. doi:10.1016/j.jviromet.2013.07.040

Silva EA, Otsuki K, Leite ACB et al (2002) HTLV-II infection associated with a chronic neurodegenerative disease: Clinical and molecular analysis. J Med Virol 257:253-257. doi:10.1002/jmv. 2138

Silva MTT, Harab RC, Leite AC et al (2007) Human T lymphotropic virus type 1 (HTLV-1) proviral load in asymptomatic carriers, HTLV-1-associated myelopathy/tropical spastic paraparesis, and other neurological abnormalities associated with HTLV-1 infection. Clin Infect Dis 44:689-692. doi:10.1086/510679

Sofian M, Aghakhani A, Farazi AA et al (2012) Serum profile of T helper 1 and $\mathrm{T}$ helper 2 cytokines in hepatitis $\mathrm{C}$ virus infected patients. Hepat Mon 12:e6156. doi:10.5812/hepatmon.6156 